\title{
THE ALGEBRAIC STRUCTURE OF LEFT SEMI-TRUSSES
}

\author{
I. COLAZZO AND A. VAN ANTWERPEN
}

\begin{abstract}
The distributive laws of ring theory are fundamental equalities in algebra. However, recently in the study of the Yang-Baxter equation, many algebraic structures with alternative "distributive" laws were defined. In an effort to study these "left distributive" laws and the interaction they entail on the algebraic structures, Brzeziński introduced skew left trusses and left semi-trusses. In particular the class of left semi-trusses is very wide, since it contains all rings, associative algebras and distributive lattices. In this paper, we investigate the subclass of left semi-trusses that behave like the algebraic structures that came up in the study of the Yang-Baxter equation. We study the interaction of the operations and what this interaction entails on their respective semigroups. In particular, we prove that in the finite case the additive structure is a completely regular semigroup. Secondly, we apply our results on a particular instance of a left semi-truss called an almost left semi-brace, introduced by Miccoli to study its algebraic structure. In particular, we show that one can associate a left semi-brace to any almost left semi-brace. Furthermore, we show that the set-theoretic solutions of the Yang-Baxter equation originating from almost left semi-braces arise from this correspondence.
\end{abstract}

\section{INTRODUCTION}

The classical examples of a set carrying two operations that interact with each other are rings, associative algebras and lattices. In recent years, several new classes have been defined and actively studied. In the process of studying the Yang-Baxter equation, Rump defined braces in [23. A left brace, following the equivalent definition of Cedó, Jespers and Okniński [12], is a set $B$ with an abelian group structure $(B,+)$ and another group structure $(B, \circ)$ such that, for any $a, b, c \in B$, it holds that $a \circ(b+c)=(a \circ b)-a+(a \circ c)$, where $-a$ denotes the inverse of $a$ in $(B,+)$. It was shown that left braces provide involutive, non-degenerate set-theoretic solutions of the Yang-Baxter equation. Recall, a set-theoretic solution of the Yang-Baxter equation is a tuple $(X, r)$, where $X$ is a set and $r: X \times X \longrightarrow X \times X$ a map such that

$$
\left(r \times \operatorname{id}_{X}\right)\left(\operatorname{id}_{X} \times r\right)\left(r \times \operatorname{id}_{X}\right)=\left(\operatorname{id}_{X} \times r\right)\left(r \times \operatorname{id}_{X}\right)\left(\operatorname{id}_{X} \times r\right) .
$$

Denote $r(x, y)=\left(\lambda_{x}(y), \rho_{y}(x)\right)$. Then, $r$ is called left (resp. right) non-degenerate if $\sigma_{x}$ (resp. $\left.\gamma_{x}\right)$ is bijective for every $x \in X$. The introduction of left braces 223 provided a novel algebraic way to describe all involutive, nondegenerate set-theoretic solutions, which was a problem posed by Drinfel'd [15] in 1992. In the wake of this discovery, the notion of a skew left brace was introduced by Guarnieri and Vendramin [16]. A skew left brace is a set $B$ equipped with two group operations $(B,+)$ and $(B, \circ)$, where, for any $a, b, c \in B$, it holds that $a \circ(b+c)=(a \circ b)-a+(a \circ c)$. Skew left braces provide bijective non-degenerate set-theoretic solutions of the Yang-Baxter equation and vice versa. Hence, the structure of (skew) left braces has been intensively studied (see, among others, [1, 10, 11, 17, 21]). In particular, several authors have used ring and group theoretical methods to study these structures [1, 14, 17, 18, 20. However, the introduction of these techniques raises several intriguing questions. Can these methods be applied in a completely general algebraic framework? What is the effect of a "left distributive" law? With these questions in mind Brzeziński introduced skew left trusses [3] and left semi-trusses [2. A left semi-truss is a set $B$ equipped with two semigroups $(B,+)$ and $(B, \circ)$ and for every $a \in B$ there exists a map $\lambda_{a}$ such that for any $b, c \in A$, it holds that

$$
a \circ(b+c)=(a \circ b)+\lambda_{a}(c) .
$$

If both $(B,+)$ and $(B, \circ)$ are groups, then $(B,+, \circ, \lambda)$ is called a skew left truss. This general framework encompasses the classes of rings, algebras and even distributive lattices. As both skew left trusses and left semi-trusses are concrete objects, an algebraic study is possible and warranted. Recently, Brzeziński [4 and Brzeziński and Rybołowicz [5, 6] have introduced and applied a module theory for left trusses in a succesful effort to study both ring and brace theoretical modules in the same framework.

2010 Mathematics Subject Classification. Primary:16T25; Secondary: 16Y99, 20M17, 81R50.

Key words and phrases. Quantum Yang-Baxter equation, Set-theoretic solution, Brace, Semi-brace. 
As most theory and knowledge originates from understanding examples, Brzeziński [2] has studied several subclasses of left semi-trusses. In particular, those where the additive semigroup is either left cancellative or an inverse semigroup. In this paper, we continue this study and restrict ourselves to the subclass of brace-like left semitrusses. In particular, these comprise all (skew) left braces, left semi-braces [7, 19] and almost left semi-braces [22]. As an application of our results, we study the class of almost left semi-braces, which was introduced by Miccoli [22. In particular, we show that the additive semi-group of an almost left semi-brace is completely simple and associate to every almost left semi-brace a left semi-brace. Furthermore, as the Yang-Baxter equation is being actively researched, we show that the solution one can associate with an almost left semi-brace is already the associated solution of a left semi-brace. In particular, this shows that this path of generalization will not yield a universal algebraic structure that produces set-theoretic solutions, which is interesting and useful knowledge in its own right. However, several other possibilities for such a structure remain, as evidenced by the recent study of left non-degenerate solutions and their structure monoid by Cedó, Jespers and Verwimp [13] and generalized semi-braces by Catino, Colazzo and Stefanelli 8$]$.

\section{Preliminaries}

Brzeziński in [3] introduced skew left trusses to deepen the understanding of the nature of the brace-distributive law.

Definition 1. A set $B$ equipped with a group structure $(B,+)$ and group structure $(B, \circ)$ is called a skew left truss $(B,+, \circ, \sigma)$, if there exists a map $\sigma: B \longrightarrow B$ such that, for any $a, b, c \in B$, it holds that

$$
a \circ(b+c)=(a \circ b)-\sigma(a)+(a \circ b),
$$

where $-x$ denotes the inverse of $x$ in $(B,+)$.

Example 2. Every skew left brace $(B,+, \circ)$ is a skew left truss $\left(B,+, \circ, \mathrm{id}_{B}\right)$.

Example 3. Let $(G,+)$ be a centerless group. For any $a, b \in G$, denote $a \circ b=a+b-a$. Then, $(G, \circ) \cong(G,+)$ is a group. Furthermore, for any $a, b, c \in G$, it holds that

$$
a \circ(b+c)=a+b+c-a=a+b-a+a+c-a=a \circ b+a \circ c .
$$

Hence, $(G,+, \circ$, id $)$ is a skew left truss.

In particular, the class of skew left semi-trusses contains all skew left braces. Note that Brzeziński has extended his notion of skew left trusses such that the additive group $(B,+)$ is replaced by a heap $[3]$. For the continuity of the exposition, we chose to present the definition above.

Later, Brzeziński [2] extended this to the notion of left semi-trusses.

Definition 4. Let $B$ be a set with two associative operations $(B,+)$ and $(B, \circ)$. If, for any $a \in B$, there exists a map $\lambda_{a}: B \longrightarrow B$ such that for any $b, c \in B$, it holds that

$$
a \circ(b+c)=(a \circ b)+\lambda_{a}(b),
$$

then $(B,+, \circ, \lambda)$ is called a left semi-truss.

Clearly, the class of semi-trusses contains all skew left trusses, rings, associative algebras and distributive lattices. This entails that it will prove difficult to present deep results on this class. However, one may examine large subclasses in an effort to gain a deeper understanding of the larger phenomenon. In particular, Brzeziński 2] focused on semi-trusses with $(B,+)$ a left cancellative or inverse semigroup. He showed that in these cases, for many semigroups, the "distributive" law can be rewritten in a fashion that closely resembles the "distributive" law of skew left braces.

In this paper, we examine the effect of the "distributive" law on the interaction between the additive and multiplicative structures of the left semi-truss. The following example shows that one will have to restrict to interesting subclasses. In particular, it shows that every semigroup can occur as the additive or multiplicative semigroup of a left semi-truss.

Example 5. Let $(S,+)$ be any semigroup. Then, $\left(S,+,+, \mathrm{id}_{S}\right)$ is a left semi-truss.

Example 6. Let $(B,+)$ be a group. For any $a, b \in B$, denote $a \circ b=a+b-a$. Then, $(B,+, \circ$, id $)$ is a left semi-truss.

We examine more closely the class of left semi-trusses that resembles (skew) left braces. 
Definition 7. Let $B$ be a set with two operations + and $\circ$ such that $(B,+)$ is a semigroup, $(B, \circ)$ a group, and $\lambda: B \rightarrow$ End $(B,+)$ a morphism from $(B, \circ)$ into the endomorph of $(B,+)$. We say that $(B,+, \circ, \lambda)$ is a brace-like left semi-truss if, for all $a, b, c \in B$, the following condition holds

$$
a \circ(b+c)=a \circ b+\lambda_{a}(c) .
$$

We call $(B,+)$ the additive semigroup, and $(B, \circ)$ the multiplicative group of the brace-like left semi-truss $(B,+, \circ, \lambda)$.

The class we restrict to contains all left semi-braces [7, 19. Recall that a (left cancellative) left semi-brace is a triple $(B,+, \circ)$ such that $(B,+)$ is a (left cancellative) semigroup, $(B, \circ)$ is a group and, for all $a, b, c \in B$, the following condition holds

$$
a \circ(b+c)=a \circ b+a \circ(\bar{a}+c),
$$

where $\bar{a}$ denotes the inverse of $a$ in $(B, \circ)$. If $(B,+, \circ)$ is a left semi-brace, then it is a brace-like left semi-truss with $\lambda_{a}(b)=a \circ(\bar{a}+b)$, for every $a, b \in B$, since by [7. Proposition 3] and [19, Lemma 2.12] the function $\lambda: B \rightarrow$ End $(B,+), a \rightarrow \lambda_{a}$ is a morphism. Vice versa if $(B,+, \circ, \lambda)$ is a brace-like left semi-truss with $\lambda_{a}(b)=a \circ(\bar{a}+b)$, then $(B,+, \circ)$ is a left semi-brace.

Let $(B,+, \circ, \lambda)$ be a brace-like left semi-truss. The element 1 denotes the identity of the multiplicative group $(B, \circ)$. First, we should remark that one will not be able to give restrictions on the properties of the group $(B, \circ)$ of a brace-like semi-truss.

Example 8. Let $(B, \circ)$ be any group. Denote for any $a, b \in B$, the operation $a+b=a$, i.e. $(B,+)$ is a left zero semigroup. Then, consider any semigroupmorphism $\lambda:(B, \circ) \longrightarrow \operatorname{Map}(B, B)$. Then, $(B,+, \circ, \lambda)$ is a brace-like left semi-truss.

However, in the next Section we shall prove that there does exist a restriction on the additive semigroup $(B,+)$.

\section{The Algebraic StruCture of A SEMI-TRUsS}

In this section, we focus on the additive structure of a brace-like left semi-truss $B$. In particular, we prove that if $B$ is finite, then the additive semigroup $(B,+)$ is a completely simple semigroup.

Lemma 9. Let $(B,+, \circ, \lambda)$ be a brace-like left semi-truss and 1 be the identity of $(B, \circ)$. The following statements hold:

1. $\lambda_{1} \lambda_{1}=\lambda_{1}$

2. $\lambda_{1} \lambda_{a}=\lambda_{a}=\lambda_{a} \lambda_{1}$, for any $a \in B$;

3. $a+b=a+\lambda_{1}(b)$, for all $a, b \in B$. In particular $B+B=B+\lambda_{1}(B)$;

4. $1+B$ is a subsemigroup of $(B, \circ)$.

Proof. Since $\lambda$ is a morphism and $(B, \circ)$ is a group with identity 1 , both 1 . and 2. easily follow.

3. Let $a, b \in B$. Then

$$
a+b=1 \circ(a+b)=1 \circ a+\lambda_{1}(b)=a+\lambda_{1}(b) .
$$

4. Let $a, b \in B$. Then

$$
(1+a) \circ(1+b)=(1+a) \circ 1+\lambda_{(1+a)}(b)=1+a+\lambda_{(1+a)}(b) \in 1+B .
$$

Therefore, $1+B$ is a subsemigroup of $(B, \circ)$.

Lemma 10. Let $(B,+, \circ, \lambda)$ be a brace-like left semi-truss. If $B$ has at least two elements then $(B,+)$ does not contain a zero element.

Proof. Suppose $\theta$ is a zero element of $(B,+)$. Then, for all $a, b \in B$, it holds that

$$
a \circ \theta=a \circ(\bar{a} \circ \theta+\theta)=a \circ \bar{a} \circ \theta+\lambda_{a}(\theta)=\theta+\lambda_{a}(\theta)=\theta .
$$

As $(B, \circ)$ is a group, it follows that $B=\{\theta\}$, a contradiction.

Lemma 11. Let $(B,+, \circ, \lambda)$ be a brace-like left semi-truss and 1 be the identity of $(B, \circ)$. The following statements hold:

1. $1 \in B+B$ and $B+B$ is a subgroup of the multiplicative group $(B, \circ)$; 
2. $(B \backslash(B+B)) \cup\{1\}$ is a subgroup of $(B, \circ)$;

3. $B=B+B$.

Proof.

1. Let $a, b \in B$. Then

$$
1=\overline{a+b} \circ(a+b)=\overline{a+b} \circ a+\lambda_{\overline{a+b}}(b) \in B+B .
$$

Moreover, let $b, c \in B$. Then

$$
\overline{a+b} \circ(c+d)=\overline{a+b} \circ c+\lambda \overline{a+b}(d) \in B+B .
$$

Hence $(B+B, \circ)$ is a subgroup of $(B, \circ)$.

2. Put $D:=(B \backslash(B+B)) \cup\{1\}$. We need to show that $\bar{a} \circ b \in D$, for all $a, b \in D$. Suppose the contrary, i.e., assume there exist distinct $a, b \in D$ such that $\bar{a} \circ b=s+t$, for some $s, t \in B$. Hence

$$
b=a \circ(s+t)=a \circ s+\lambda_{a}(t) \in B+B,
$$

which is a contradiction.

3. The claim is trivially true if $B=\{1\}$. Suppose $|B| \geq 2$. From 1. and 2., we know that the group $(B, \circ)$ is the union of two subgroups $B+B$ and $(B \backslash(B+B)) \cup\{1\}$. Because the intersection of these groups is $\{1\}$ it follows that $B=B+B$ or $B+B=\{1\}$. In the second case, $b+1=1=1+b$, for any $b \in B$, in contradiction with Lemma 10 , since $|B| \geq 2$.

Lemma 12. Let $(B,+, \circ, \lambda)$ be a brace-like left semi-truss with $B$ finite. Then there exists an idempotent element $z$ of $(B,+)$ such that $z+B+z=1+B+z$ is a subgroup of $(B,+)$.

Proof. For any $a \in B$, define $a^{1}:=a$, for every integer $n \geq 1$, and $a^{n+1}=a \circ a^{n}$.

First, suppose that for all $b \in B, 1+b=1$. Then, in particular, $1+1=1$, i.e., 1 is an idempotent element. Moreover, $1+B+1=\{1\}$ and the claim is trivially true.

Otherwise, since $B$ is finite, for any $b \in B$ such that $1+b \neq 1$, there exists an integer $n_{b} \geq 2$ such that $(1+b)^{n_{b}}=1$. Then

$$
\begin{aligned}
1 & =(1+b)^{n_{b}-1} \circ(1+b) \\
& =(1+b)^{n_{b}-1}+\lambda_{(1+b)^{n_{b}-1}}(b) .
\end{aligned}
$$

Continuing, we obtain inductively that

$$
1=1+b+\sum_{i=1}^{n_{b}-1} \lambda_{(1+b)^{i}}(b) .
$$

Set $z_{b}:=b+\sum_{i=1}^{n_{b}-1} \lambda_{(1+b)^{i}}(b)$. Then $1+z_{b}=1$.

First, if $z_{b}=1$, then 1 is idempotent and it is the identity of $1+B+1$. Moreover, since $B$ is finite, for any $a \in B$ such that $1+a+1 \neq 1$, there exists a integer $k \geq 2$, such that $(1+a+1)^{k}=1$. Set $\underline{a}:=\sum_{i=1}^{k-1} \lambda_{(1+a+1)^{i}}(a+1)$, then

$$
\begin{aligned}
(1+a+1)+(1+\underline{a}+1) & =1+a+1+\sum_{i=1}^{k-1} \lambda_{(1+a+1)^{i}}(a+1)+1 \\
& =(1+a+1)^{k}+1=1+1=1,
\end{aligned}
$$

i.e., every element in $1+B+1$ has a right inverse. Therefore $1+B+1$ is a group. Otherwise, $z_{b} \neq 1$. Since $B$ is finite, there exists an integer $m_{b} \geq 2$, such that $z_{b}^{m_{b}}=1$ and, so,

$$
\lambda_{z_{b}}^{m_{b}}=\underbrace{\lambda_{z_{b}} \lambda_{z_{b}} \ldots \lambda_{z_{b}}}_{m_{b} \text { times }}=\lambda_{1} .
$$

Moreover, note that $z_{b}=z_{b} \circ 1=z_{b} \circ\left(1+z_{b}\right)=z_{b}+\lambda_{z_{b}}\left(z_{b}\right)$, then

$$
z_{b}=z_{b}+\lambda_{z_{b}}\left(z_{b}\right)=z_{b}+\lambda_{z_{b}}\left(z_{b}+\lambda_{z_{b}} z_{b}\right)=z_{b}+\lambda_{z_{b}}\left(z_{b}\right)+\lambda_{z_{b}}^{2}\left(z_{b}\right) .
$$


If we continue this inductively, we obtain that

$$
z_{b}=z_{b}+\lambda_{z_{b}}\left(z_{b}\right)+\cdots+\lambda_{z_{b}}^{m_{b}-1}(z)+\lambda_{z_{b}}^{m_{b}}\left(z_{b}\right)+\cdots+\lambda_{z_{b}}^{2 m_{b}-1}\left(z_{b}\right) .
$$

Set $\underline{z_{b}}:=z_{b}+\lambda_{z_{b}}\left(z_{b}\right)+\cdots+\lambda_{z_{b}}^{m_{b}-1}\left(z_{b}\right)$, then

$$
z_{b}=\underline{z_{b}}=\underline{z_{b}}+\lambda_{z_{b}}^{m_{b}}\left(\underline{z_{b}}\right)=\underline{z_{b}}+\lambda_{1}\left(\underline{z_{b}}\right)=\underline{z_{b}}+\underline{z_{b}}=z_{b}+z_{b} .
$$

In particular, this shows that $z_{b}$ is an idempotent. From now on, we focus on $b \in 1+B$. Then, by definition, there exists $z_{b}^{\prime}$ such that $z_{b}=1+z_{b}^{\prime}$. Then

$$
\begin{aligned}
1 & =z_{b}^{m_{b}}=\left(1+z_{b}^{\prime}\right)^{m_{b}}=1+z_{b}^{\prime}+\sum_{i=1}^{m_{b}-1} \lambda_{z_{b}^{i}}\left(z_{b}^{\prime}\right)=z_{b}+\sum_{i=1}^{m_{b}-1} \lambda_{z_{b}^{i}}\left(z_{b}^{\prime}\right) \\
& =z_{b}+\left(z_{b}+\sum_{i=1}^{m_{b}-1} \lambda_{z_{b}^{i}}\left(z_{b}^{\prime}\right)\right)=z_{b}+z_{b}^{m_{b}} \\
& =z_{b}+1 .
\end{aligned}
$$

Finally, we prove that $1+B+z_{b}$, with $b \in 1+B$, is a subgroup of $(B,+)$ with identity $z_{b}$. First,

$$
z_{b}=z_{b}+z_{b}=1+z_{b}^{\prime}+z_{b} \in 1+B+z_{b}
$$

Moreover, for all $a \in B$,

$$
\left(1+a+z_{b}\right)+z_{b}=1+a+z_{b}
$$

and

$$
z_{b}+\left(1+a+z_{b}\right)=\left(z_{b}+1\right)+a+z_{b}=1+a+z_{b} .
$$

Furthermore, since $B$ is finite for all $a \in B$, there exists a positive integer $k$ such that $\left(1+a+z_{b}+1\right)^{k}=1$. If $k=1$, then $\left(1+a+z_{b}\right)+\left(1+z_{b}^{\prime}+z_{b}\right)=1+z_{b}^{\prime}+z_{b}=z_{b}+z_{b}=z_{b}$, otherwise

$$
1=\left(1+a+z_{b}+1\right)^{k}=1+a+z_{b}+1+\sum_{i=1}^{k-1} \lambda_{\left(1+a+z_{b}+1\right)^{i}}\left(a+z_{b}+1\right),
$$

set $\underline{a}:=\sum_{i=1}^{k-1} \lambda_{\left(1+a+z_{b}+1\right)^{i}}\left(a+z_{b}+1\right)$, then

$$
\left(1+a+z_{b}\right)+\left(1+\underline{a}+z_{b}^{\prime}+z_{b}\right)=\left(1+a+z_{b}+1+\underline{a}\right)+z_{b}^{\prime}+z_{b}=1+z_{b}^{\prime}+z_{b}=z_{b}+z_{b}=z_{b},
$$

i.e., every element in $1+B+z_{b}$ has a right inverse. Therefore $1+B+z_{b}$ is a group. Finally, for any $a \in B$, $1+a+z_{b}=z_{b}+1+a+z_{b} \in z_{b}+B+z_{b}$ and $z_{b}+a+z_{b}=1+z_{b}^{\prime}+a+z_{b} \in 1+B+z$. Hence, $z_{b}+B+z_{b}=1+B+z_{b}$.

Lemma 13. Let $(B,+, \circ, \lambda)$ be a brace-like left semi-truss. If there exists an idempotent element $z$ of $(B,+)$ such that $z+B+z$ is a subgroup of $(B,+)$. Then, for any $c \in B, c \in c+z+B$ and, in particular, $B+z+B=B$.

Proof. Let $c \in B$. If $d \in B$ is such that $z+d+z$ is the inverse of $z+\lambda_{z} \lambda_{\bar{c}}(z)+z$ in $z+B+z$ and $b:=\lambda_{c} \lambda_{\bar{z}}(z+d+z)$. Then $c=c+z+b$. Indeed

$$
\begin{aligned}
c & =c \circ \bar{z} \circ z=c \circ \bar{z} \circ\left(z+\lambda_{z} \lambda_{\bar{c}}(z)+z+z+d+z\right) \\
& =c \circ \bar{z} \circ\left(z+\lambda_{z} \lambda_{\bar{c}}(z)+\lambda_{z} \lambda_{\bar{c}}\left(\lambda_{c} \lambda_{\bar{z}}(z+d+z)\right)\right) \\
& =c \circ \bar{z} \circ\left(z+\lambda_{z} \lambda_{\bar{c}}(z+b)\right) \\
& =c \circ \bar{z} \circ z \circ \bar{c} \circ(c+z+b) \\
& =c+z+b \in B+z+B .
\end{aligned}
$$

Therefore $B=B+z+B$.

Theorem 14. Let $(B,+, \circ, \lambda)$ be a brace-like left semi-truss. If there exists an idempotent element $z$ of $(B,+)$ such that $z+B+z$ is a subgroup of $(B,+)$. Then $z$ is a primitive idempotent and $(B,+)$ is completely simple semigroup. In particular, this holds if $B$ is finite. 
Proof. Let $b \in B$. Since $z+B+z$ is a group, there exists an element $\underline{b} \in B$ such that $z+b+z+z+\underline{b}+z=z$. Moreover, by Lemma 13] for any $c \in B$, there exists an element $d \in B$ such that $c=c+z+d$. Then

$$
c=c+z+d=(c+z)+b+(z+z+\underline{b}+z+d) \in B+b+B .
$$

Hence, $B=B+b+B$. Therefore, every principal ideal, and thus every ideal of $B$ is trivial, i.e., $(B,+)$ is a simple semigroup. Since $z+B+z$ is a subgroup of $(B,+)$, then $z$ is a primitive idempotent of $(B,+)$. Hence $(B,+)$ is a completely simple semigroup.

Theorem 14 shows that the role of idempotents of $(B,+)$ should not be underestimated. Hence, it is an interesting question whether these idempotents form a subsemi-truss, like in the case for left semi-braces [7, 19]. Denote the set of idempotents of $(B,+)$ by $E(B)$. The following lemma proves that $E(B)$ is closed under any $\lambda_{a}$, for each left semi-truss $(B,+, \circ, \lambda)$.

Lemma 15. Let $(B,+, \circ, \lambda)$ be a left semi-truss. For any $a \in B, \lambda_{a}(E(B)) \subseteq E(B)$.

Proof. If $e \in E(B)$, then $\lambda_{a}(e)=\lambda_{a}(e+e)=\lambda_{a}(e)+\lambda_{a}(e)$. Hence, $\lambda_{a}(e) \in E(B)$.

However, in general $E(B)$ is not closed with respect to $\circ$ as shown by the following example.

Example 16. Let $B$ be the Klein group defined by

$$
B:=\left\langle a, b \mid a^{2}=b^{2}=(a b)^{2}=1\right\rangle .
$$

Every element $x \in B$ can be represented by $x=a^{i} b^{j}$ with $i, j \in \mathbb{Z}$. Clearly, two elements $a^{i} b^{j}$ and $a^{h} b^{k}$ are equal if and only if $i \equiv h \bmod 2$ and $j \equiv k \bmod 2$. Define

$$
a^{i} b^{j}+a^{h} b^{k}:=a^{1+i+h} b^{j}
$$

and

$$
\lambda: B \longrightarrow \text { End }(B,+), \quad a^{i} b^{j} \longmapsto f
$$

where $f: B \rightarrow B, a^{h} b^{k} \mapsto a^{h}$. Then $(B,+, \circ, \lambda)$ is a left semi-truss. Indeed

$$
\begin{gathered}
\lambda_{a^{i} b^{j}} \lambda_{a^{h} b^{k}}\left(a^{y} b^{z}\right)=f f\left(a^{y} b^{z}\right)=f\left(a^{y}\right)=a^{y}=f\left(a^{y} b^{z}\right)=\lambda_{a^{i} b^{j} \circ a^{h} b^{k}}\left(a^{y} b^{z}\right), \\
\lambda_{a^{i} b^{j}}\left(a^{h} b^{k}+a^{y} b^{z}\right)=\lambda_{a^{i} a^{j}}\left(a^{1+h+y} b^{k}\right)=a^{1+h+y}
\end{gathered}
$$

and

$$
\lambda_{a^{i} b^{j}}\left(a^{h} b^{k}\right)+\lambda_{a^{i} b^{j}}\left(a^{y} b^{z}\right)=a^{h}+a^{y}=a^{1+h+y} .
$$

Moreover

$$
a^{i} b^{j} \circ\left(a^{h} b^{k}+a^{y} b^{z}\right)=a^{i} b^{j} \circ\left(a^{1+h+y} b^{k}\right)=a^{i+1+h+y} b^{j+k}
$$

and

$$
a^{i} b^{j} \circ a^{h} b^{k}+\lambda_{a^{i} b^{j}}\left(a^{y} b^{z}\right)=a^{i+h} b^{j+k}+a^{y}=a^{1+i+h+y} b^{j+k} .
$$

Finally, $a+a=a$, i.e., $a \in E(B)$, but $a \circ a=1$ and $1+1=a$, i.e., $a \circ a \notin E(B)$.

In particular, this shows that in general the additive and multiplicative semigroups of a brace-like left semi-truss fit together very differently than those of a left semi-brace, where a nice decomposition theorem [7, 19] can be obtained. 


\section{Almost Left SEMi-BRACE AND SOlutions}

In this section, we focus on a particular instance of brace-like left semi-trusses called almost left semi-braces. Our definition extends the one introduced by Miccoli [22, where one works under the restriction that the semigroup $(B,+)$ is left cancellative and, for all $a, b \in B$, it holds

$$
(\iota(a)+b) \circ \iota(1)=\iota(a)+b \circ \iota(1)
$$

where 1 is the identity of the group $(B, \circ)$.

Definition 17. Let $B$ be a set with two operations + and $\circ$ such that $(B,+)$ is a semigroup, $(B, \circ)$ is a group, and $\iota: B \rightarrow B$ is a map such that, for all $a, b \in B$, the following condition holds

$$
\iota(a \circ b)=\bar{b} \circ \iota(a),
$$

where $\bar{b}$ denotes the inverse of $b$ in $(B, \circ)$. We say that $(B,+, \circ, \iota)$ is an almost left semi-brace if, for all $a, b, c \in B$, it holds

$$
a \circ(b+c)=a \circ b+a \circ(\iota(a)+c) .
$$

We call $(B,+)$ the additive semigroup, and $(B, \circ)$ the multiplicative group of the almost left semi-brace $(B,+, \circ, \iota)$.

If $(B,+, \circ)$ is a left semi-brace, then it is an almost left semi-brace with $\iota: B \rightarrow B$ defined by $\iota(a)=\bar{a}$, for any $a \in B$. Conversely, if $(B,+, \circ, \iota)$ is an almost semi-brace such that $\iota(a)=\bar{a}$, then $(B,+, \circ)$ is a left semi-brace.

The following lemma proves that given an almost left semi-brace $(B,+, \circ, \iota)$, then it is possible to define a morphism $\lambda$ such that $(B,+\circ, \lambda)$ is a brace-like left semi-truss.

Lemma 18. Let $(B,+, \circ, \iota)$ be an almost left semi-brace. For any $a \in B$, it follows that $\lambda_{a} \in$ End $(B,+)$, where $\lambda_{a}(b):=a \circ(\iota(a)+b)$, for every $b \in B$. Moreover, $\lambda:(B, \circ) \rightarrow$ End $(B,+), a \mapsto \lambda_{a}$ is a homomorphism and, for any $a \in B$, it holds that $\lambda_{a}(E(B)) \subseteq E(\iota(1)+B)$.

Proof. First, note that defining, for all $a, b \in B, \lambda_{a}(b):=a \circ(\iota(a)+b)$, (4) is clearly equivalent to

$$
a \circ(b+c)=a \circ b+\lambda_{a}(c) .
$$

Let $a, b, x, y \in B$. By (5) it holds that

$$
\begin{aligned}
\lambda_{a}(x+y) & =a \circ(\iota(a)+x+y) \\
& =a \circ(\iota(a)+x)+\lambda_{a}(y) \\
& =\lambda_{a}(x)+\lambda_{a}(y)
\end{aligned}
$$

and, by (3) and (5), it follows that

$$
\begin{aligned}
\lambda_{a \circ b}(x) & =a \circ b \circ(\iota(a \circ b)+x) \\
& =a \circ b \circ(\bar{b} \circ \iota(a)+x) \\
& =a \circ\left(b \circ \bar{b} \circ \iota(a)+\lambda_{b}(x)\right) \\
& =a \circ\left(\iota(a)+\lambda_{b}(x)\right) \\
& =\lambda_{a} \lambda_{b}(x) .
\end{aligned}
$$

Moreover, if $e \in E(B)$, then $\lambda_{a}(e)=\lambda_{a}(e+e)=\lambda_{a}(e)+\lambda_{a}(e)$. Hence, $\lambda_{a}(e) \in E(B)$. Furthermore,

$$
\iota(1)+\lambda_{a}(e)=a \circ \bar{a} \circ \iota(1)+\lambda_{a}(e)=a \circ \iota(a)+\lambda_{a}(e)=a \circ(\iota(a)+e)=\lambda_{a}(e) .
$$

Therefore, $\lambda_{a}(E(B)) \subseteq E(\iota(1)+B)$.

Hence, if $(B,+, \circ, \iota)$ is an almost left semi-brace, defining $\lambda:(B, \circ) \rightarrow$ End $(B,+)$ as in Lemma 15, then $(B,+, \circ, \lambda)$ is a brace-like left semi-truss. But not all brace-like left semi-trusses are almost left semi-braces. Just consider the following example to be convinced.

Example 19. Let $(B, \circ)$ be a group, $f: B \rightarrow B$ a map such that $f^{2}=f$ and denote $(B,+)$ the left zero semigroup on the set $B$. Then $(B,+, \circ, \lambda)$ where $\lambda: B \rightarrow \operatorname{End}(B,+), a \mapsto f$ is a brace-like left semi-truss, but, in general, is not an almost semi-brace. For instance, suppose that $f=\operatorname{id}_{B}$ and there exists $\iota: B \rightarrow B$ such $\lambda_{a}(b)=a \circ(\iota(a)+b)$. Hence, $b=\lambda_{a}(b)=a \circ \iota(a)$, for any $a, b \in B$. In particular, for $b=a$, we get $\iota(a)=1$. Therefore, $b=\lambda_{a}(b)=a$, for any $a, b \in B$, a contradiction, if $|B|>1$. 
If $\left(B_{1},+_{1}, \circ_{1}, \iota_{1}\right)$ and $\left(B_{2},+_{2}, \circ_{2}, \iota_{2}\right)$ are almost left semi-braces then a map $f: B_{1} \rightarrow B_{2}$ is a homomorphism of almost left semi-braces if $f$ is a semigroup homomorphism from $\left(B_{1},+_{1}\right)$ to $\left(B_{2},+_{2}\right)$, $f$ is a group homomorphism from $\left(B_{1}, \circ_{1}\right)$ to $\left(B_{2}, \circ_{2}\right)$ and, $f \iota_{1}=\iota_{2} f$. Hence, a left semi-brace $(B,+, \circ)$ considered as an almost left semi-brace can not be isomorphic to an almost left semi-brace $\left(B,+, \circ, \iota_{B}\right)$ with $\iota_{B}(1) \neq 1$. Indeed, such an isomorphism $f$ has to satisfy $\iota_{B}(1)=\iota_{B} f(1)=f(1)=1$. However, we can associate a left semi-brace to any almost left semi-brace. To this purpose, we make the following preliminary result.

Lemma 20. Let $(B,+, \circ, \iota)$ be an almost left semi-brace. The following properties hold.

1. $a+b=a+\lambda_{1}(b)=a+\iota(1)+b$, for all $a, b \in B$. In particular $B+B=B+\iota(1)+B$

2. $\iota$ is bijective and $\iota(a)=\bar{a} \circ \iota(1)$, for every $a \in B$.

Proof.

1. Let $a, b \in B$. Then, by Lemma 9.3 and Lemma $18, a+b=a+\lambda_{1}(b)$ and

$$
a+b=a+\lambda_{1}(b)=a+1 \circ(\iota(1)+b)=a+\iota(1)+b .
$$

2. Let $a \in B$. By (3) in Definition [17, we get

$$
\iota(a)=\iota(1 \circ a)=\bar{a} \circ \iota(1) .
$$

Furthermore, $\iota$ is bijective. Indeed, if $a, b \in B$, such that $\iota(a)=\iota(b)$, then by (6) $\bar{a} \circ \iota(1)=\bar{b} \circ \iota(1)$ and so $a=b$. Furthermore, if $b \in B$, then by (6),

$$
\iota(\iota(1) \circ \bar{b})=b \circ \overline{\iota(1)} \circ \iota(1)=b \circ 1=b .
$$

Applying Theorem 14 to almost left semi-braces, we obtain the following.

Proposition 21. Let $(B,+, \circ, \iota)$ be a finite almost left semi-brace. Then $(B,+)$ is a completely simple semigroup, where 1 is a primitive idempotent.

Now, let $(B,+, \circ, \iota)$ be an almost left semi-brace. Define the following operation on $B$ :

$$
\oplus: B \times B \longrightarrow B, \quad(a, b) \longmapsto \iota^{-1}(\iota(a)+\iota(b))
$$

Note that, from (3) , if $(B,+, \circ, \iota)$ is an almost left semi-brace, then it follows that

$$
\iota^{-1}(a \circ b)=\iota^{-1}(b) \circ \bar{a}
$$

for all $a, b \in B$.

Proposition 22. If $(B,+, \circ, \iota)$ is an almost left semi-brace and $\oplus$ defined as above, then $\left(B, \oplus, \circ^{o p}\right)$ is a left semi-brace, called left semi-brace associated to the almost left semi-brace $(B,+, \circ, \iota)$.

Proof. Clearly $\left(B, \circ^{o p}\right)$ is a group. Moreover, if $a, b, c, \in B$, then

$$
(a \oplus b) \oplus c=\iota^{-1}(\iota(a)+\iota(b)) \oplus c=\iota^{-1}(\iota(a)+\iota(b)+\iota(c))
$$

and

$$
a \oplus(b \oplus c)=a \oplus \iota^{-1}(\iota(b)+\iota(c))=\iota^{-1}(\iota(a)+\iota(b)+\iota(c)) .
$$

Thus $(B, \oplus)$ is a semigroup. Finally, if $a, b, c \in B$ then

$$
a \circ^{o p}(b \oplus c)=\iota^{-1}(\iota(b)+\iota(c)) \circ a
$$

and by (7) and (4)

$$
\begin{aligned}
a \circ^{o p} b \oplus a \circ^{o p}(\bar{a} \oplus c) & =b \circ a \oplus(\bar{a} \oplus c) \circ a=b \circ a \oplus \iota^{-1}(\iota(\bar{a})+\iota(c)) \circ a \\
& =b \circ a \oplus \iota^{-1}(\bar{a} \circ(\iota(a)+\iota(c))) \\
& =\iota^{-1}(\iota(b \circ a)+\bar{a} \circ(\iota(a)+\iota(c))) \\
& =\iota^{-1}(\bar{a} \circ \iota(b)+\bar{a} \circ(\iota(a)+\iota(c))) \\
& =\iota^{-1}(\bar{a} \circ(\iota(b)+\iota(c))) \\
& =\iota^{-1}((\iota(b)+\iota(c))) \circ a .
\end{aligned}
$$


Hence, $\left(B, \oplus, \circ^{o p}\right)$ is a left semi-brace.

As in 2, Corollary 2.7] we can associate a solution with an almost left semi-brace via its associated left semi-brace, whenever it is possible to associate a solution to such a semi-brace. But, under some assumptions, we can associate a solution directly to an almost semi-brace. In the following, we prove that these solutions are isomorphic.

Following the idea in [9], we provide a necessary and sufficient condition for obtaining a solution from an almost left semi-brace.

Theorem 23. Let $(B,+, \circ, \iota)$ be an almost left semi-brace such that (2) is satisfied, for all a,b $\in$ B. The map $r_{B}: B \times B \rightarrow B \times B$ defined by $r_{B}(a, b)=(a \circ(\iota(a)+b), \overline{(\iota(a)+b)} \circ b)$ for all $a, b \in B$ is a solution if and only if the following condition holds

$$
a+\lambda_{b}(c) \circ\left(\iota(1)+\rho_{c}(b)\right)=a+b \circ(\iota(1)+c)
$$

for all $a, b, c \in B$.

Proof. It is easily verified that $\left(B, r_{B}\right)$ is a solution if and only if, for all $a, b, c \in B$,

$$
\left(\lambda_{a} \lambda_{b}(c), \lambda_{\rho_{\lambda_{b}(c)}(a)} \rho_{c}(b), \rho_{\rho_{c}(b)} \rho_{\lambda_{b}(c)}(a)\right)=\left(\lambda_{\lambda_{a}(b)} \lambda_{\rho_{b}(a)}(c), \rho_{\lambda_{\rho_{b}(a)}(c)} \lambda_{a}(b), \rho_{c} \rho_{b}(a)\right) .
$$

Denote the first triple by $\left(s_{1}, s_{2}, s_{3}\right)$ and the second by $\left(t_{1}, t_{2}, t_{3}\right)$. Since, for all $x, y \in B$,

$$
\lambda_{x}(y) \circ \rho_{y}(x)=x \circ y
$$

holds, it follows that

$$
\begin{aligned}
s_{1} \circ s_{2} \circ s_{3} & =\lambda_{a} \lambda_{b}(c) \circ \lambda_{\rho_{\lambda_{b}(c)}(a)} \rho_{c}(b) \circ \rho_{\rho_{c}(b)} \rho_{\lambda_{b}(c)}(a) \\
& =\lambda_{a} \lambda_{b}(c) \circ \rho_{\lambda_{b}(c)}(a) \circ \rho_{c}(b) \\
& =a \circ \lambda_{b}(c) \circ \rho_{c}(b) \\
& =a \circ b \circ c
\end{aligned}
$$

and

$$
\begin{aligned}
t_{1} \circ t_{2} \circ t_{3} & =\lambda_{\lambda_{a}(b)} \lambda_{\rho_{b}(a)}(c) \circ \rho_{\lambda_{\rho_{b}(a)}(c)} \lambda_{a}(b) \circ \rho_{c} \rho_{b}(a) \\
& =\lambda_{a}(b) \circ \lambda_{\rho_{b}(a)}(c) \circ \rho_{b}(a) \\
& =\lambda_{a}(b) \circ \rho_{b}(a) \circ c \\
& =a \circ b \circ c .
\end{aligned}
$$

Thus, $s_{1} \circ s_{2} \circ s_{3}=t_{1} \circ t_{2} \circ t_{3}$.

Now, suppose that (8) holds. As by Lemma 15, $\lambda:(B, \circ) \rightarrow$ End $(B,+)$ is a homomorphism, and since (9) it follows that

$$
t_{1}=\lambda_{\lambda_{a}(b)} \lambda_{\rho_{b}(a)}(c)=\lambda_{\lambda_{a}(b) \circ \rho_{b}(a)}(c)=\lambda_{a \circ b}(c)=\lambda_{a} \lambda_{b}(c)=s_{1} .
$$

To prove that $s_{3}=t_{3}$, first note that for any $x, y, z \in B$, it holds

$$
\begin{aligned}
\rho_{z} \rho_{y}(x) & =\overline{\left(\iota \rho_{y}(x)+z\right)} \circ z \\
& =\overline{(\iota(\overline{(\iota(x)+y) \circ y})+z)} \circ z \\
& =\overline{(\bar{y} \circ \iota(\overline{\iota(x)+y})+z)} \circ z \\
& =\overline{(\bar{y} \circ(\iota(x)+y) \circ \iota(1)+z)} \circ z \\
& =\overline{(\bar{y} \circ(\iota(x)+y \circ \iota(1))+z)} \circ z \\
& =\overline{\left(\bar{y} \circ(\iota(x)+y \circ \iota(1))+\lambda_{1}(z)\right)} \circ z \\
& =\overline{\left(\bar{y} \circ(\iota(x)+y \circ \iota(1))+\lambda_{\bar{y}} \lambda_{y}(z)\right)} \circ z \\
& =\overline{\bar{y} \circ\left(\iota(x)+y \circ \iota(1)+\lambda_{y}(z)\right)} \circ z \\
& =\overline{(\iota(x)+y \circ(\iota(1)+z))} \circ y \circ z
\end{aligned}
$$

(by Lemma 20.1)

(by Lemma 18)

(by (4))

(by (4)) 
Hence, by (8) and (9), it follows that

$$
\begin{aligned}
s_{3} & =\rho_{\rho_{c}(b)} \rho_{\lambda_{b}(c)}(a) \\
& =\overline{\left(\iota(a)+\lambda_{b}(c) \circ\left(\iota(1)+\rho_{c}(b)\right)\right)} \circ \lambda_{b}(c) \circ \rho_{c}(b) \\
& =\overline{(\iota(a)+b \circ(\iota(1)+c))} \circ b \circ c \\
& =t_{3} .
\end{aligned}
$$

Moreover, since $s_{1} \circ s_{2} \circ s_{3}=t_{1} \circ t_{2} \circ t_{3}, t_{1}=s_{1}, s_{3}=t_{3}$, and $(B, \circ)$ is a group it holds $s_{2}=t_{2}$. Conversely, suppose that $\left(B, r_{B}\right)$ is a solution. In particular,

$$
\overline{\left(\iota(a)+\lambda_{b}(c) \circ\left(\iota(1)+\rho_{c}(b)\right)\right)} \circ b \circ c=s_{3}=t_{3}=\overline{(\iota(a)+b \circ(\iota(1)+c))} \circ b \circ c .
$$

Hence (8) holds, since, by Lemma 20.2, $\iota$ is bijective.

Let $(B,+, \circ, \iota)$ be an almost left semi-brace that satisfies (2) and (8). The map $r_{B}$ defined in Theorem 23 is said to be the solution associated to $B$.

Note that if $(B,+, \circ, \iota)$ is an almost left semi-brace such that (2) holds and $\rho: B \rightarrow \operatorname{Map}(B, B)$ is an antihomomorphism, then (8) is satisfied. Indeed, since $\iota$ is bijective, it holds

$$
\begin{aligned}
a+\lambda_{b}(c) \circ\left(\iota(1)+\rho_{c}(b)\right) & =\lambda_{b}(c) \circ \rho_{c}(b) \circ \overline{\rho_{\rho_{c}(b)} \rho_{\lambda_{b}(c)}\left(\iota^{-1}(a)\right)} \\
& =b \circ c \circ \overline{\rho_{\lambda_{b}(c) \circ \rho_{c}(b)}\left(\iota^{-1}(a)\right)} \\
& =b \circ c \circ \overline{\rho_{b \circ c}\left(\iota^{-1}(a)\right)} \\
& =b \circ c \circ \overline{\rho_{b} \rho_{c}\left(\iota^{-1}(a)\right)} \\
& =a+b \circ(\iota(1)+c)
\end{aligned}
$$

i.e., (8) is satisfied.

Following [12, p.105], we define a homomorphism between set theoretical solutions.

Definition 24. Let $X, Y$ be sets and let $r: X \times X \rightarrow X \times X$ and $r^{\prime}: Y \times Y \rightarrow Y \times Y$ be maps such that $r(a, b)=\left(\lambda_{a}(b), \rho_{b}(a)\right)$ and $r^{\prime}(x, y)=\left(\lambda_{a}^{\prime}(b), \rho_{b}^{\prime}(a)\right)$. A homomorphism from $(X, r)$ to $\left(Y, r^{\prime}\right)$ is a map $f: X \rightarrow Y$ such that $(f \times f) r=(f \times f) r^{\prime}$, i.e., $\left(f\left(\lambda_{a}(b)\right), f\left(\rho_{b}(a)\right)\right)=\left(\lambda_{f(a)}^{\prime}(f(b)), \rho_{f(b)}^{\prime}(f(a))\right)$, for all $a, b \in X$. If $f$ is bijective then we say that $(X, r)$ and $\left(Y, r^{\prime}\right)$ are isomorphic solutions.

In [9, Theorem 3], it is proved that for any left semi-brace $B$, the map $r_{B}: B \times B \rightarrow B \times B$ defined by $r_{B}(a, b)=$ $(a \circ(\bar{a}+b), \overline{(\bar{a}+b)} \circ b)$ is a solution if and only if

$$
a+\lambda_{b}(c) \circ\left(1+\rho_{c}(b)\right)=a+b \circ(1+c)
$$

where $\lambda_{b}(c)=b \circ(\bar{b}+c), \rho_{c}(b)=\overline{(\bar{b}+c)} \circ c$, and 1 is the identity of the group $(B,+)$.

In this theorem we prove that if $(B,+, \circ, \iota)$ satisfies (8), then $\left(B, \oplus, \circ^{o p}\right)$ satisfies (11) and that the solution associated to the almost left semi-brace and the solution associated to the left semi-brace are isomorphic.

Theorem 25. Let $(B,+, \circ, \iota)$ be an almost left semi-brace that satisfies (2) and (8), and let (B, $\oplus, \circ$ op $)$ be the left semi-brace associated with $B$. Then $\left(B, \oplus, \circ^{o p}\right)$ satisfies (11). Furthermore, if $r_{B}$ is the solution defined in Theorem [23, and $r_{B}^{\prime}$ the solution associated to the left semi-brace $\left(B, \oplus, \circ^{o p}\right)$ as defined in [9, Theorem 3], then $r_{B}$ and $r_{B}^{\prime}$ are isomorphic. 
Proof. First, we compute the maps $\lambda_{a}^{\prime}(b)=a \circ^{o p}(\bar{a} \oplus b)$ and $\rho_{b}^{\prime}(a)=\overline{(\bar{a} \oplus b)} \circ^{o p} b$, for the left semi-brace $\left(B, \oplus, \circ^{o p}\right)$. Let $a, b, c \in B$. By Lemma 20.2, (6), and (2), we have

$$
\begin{aligned}
\lambda_{a}^{\prime}(b) & =a \circ^{o p}(\bar{a} \oplus b)=\iota^{-1}(\iota(\bar{a})+\iota(b)) \circ a \\
& =\iota^{-1}(\bar{a} \circ(\iota(\bar{a})+\iota(b))) \\
& =\iota^{-1}(\bar{a} \circ(\iota(\bar{a})+b \circ \iota(1))) \\
& =\iota^{-1}(\bar{a} \circ(\iota(\bar{a})+\bar{b}) \circ \iota(1)) \\
& =1 \circ \overline{(\bar{a} \circ(\iota(\bar{a})+\bar{b}))} \\
& =\overline{\lambda_{\bar{a}}(\bar{b})} .
\end{aligned}
$$

Moreover, by Lemma 20.2 we obtain $\bar{b}=\iota(b) \circ \overline{\iota(1)}$. Using also (2), it follows that

$$
\begin{aligned}
\rho_{b}^{\prime}(a) & =\overline{(\bar{a} \oplus b)} \circ^{o p} b=b \circ \overline{\iota^{-1}(\iota(\bar{a})+\iota(b))} \\
& =b \circ \iota \iota^{-1}(\iota(\bar{a})+\iota(b)) \circ \overline{\iota(1)} \\
& =b \circ(\iota(\bar{a})+\bar{b} \circ \iota(1)) \circ \overline{\iota(1)} \\
& =b \circ(\iota(\bar{a})+\bar{b}) \circ \iota(1) \circ \overline{\iota(1)} \\
& =b \circ(\iota(\bar{a})+\bar{b}) \\
& =\overline{\overline{(\iota(\bar{a})+\bar{b})} \circ \bar{b}} \\
& =\overline{\rho_{\bar{b}}(\bar{a})} .
\end{aligned}
$$

Now, suppose that $(B,+, \circ, \iota)$ satisfies (2) and (8). By Lemma 20.2 and by (2), we have that

$$
\iota(\overline{a+b})=\left(\iota^{-1}(a)+b\right) \circ \iota(1)=\iota^{-1}(a)+b \circ \iota(1)=a+\iota(\bar{b}) .
$$

And using also (3), it follows that

$$
\begin{aligned}
a \oplus b \circ^{o p}(1 \oplus c) & =\iota^{-1}\left(\iota(a)+\iota\left(\iota^{-1}(\iota(1)+\iota(c)) \circ b\right)\right) \\
& =\iota^{-1}(\iota(a)+\bar{b} \circ(\iota(1)+\iota(c))) \\
& =\iota^{-1}(\iota(a)+\bar{b} \circ(\iota(1)+\bar{c} \circ \iota(1))) \\
& =\iota^{-1}(\iota(a)+\bar{b} \circ(\iota(1)+\bar{c}) \circ \iota(1)) \\
& =\iota^{-1}((\iota(a)+\bar{b} \circ(\iota(1)+\bar{c})) \circ \iota(1))
\end{aligned}
$$

and, by (8),

$$
\begin{aligned}
a \oplus \lambda_{a}^{\prime}(b) \circ^{o p}\left(1 \oplus \rho_{c}^{\prime}(b)\right) & =\iota^{-1}\left(\iota(a)+\iota\left(\iota^{-1}\left(\iota(1)+\iota\left(\overline{\rho_{\bar{c}}(\bar{b})}\right)\right) \circ \overline{\lambda_{\bar{b}}(\bar{c})}\right)\right) \\
& =\iota^{-1}\left(\iota(a)+\lambda_{\bar{b}}(\bar{c}) \circ\left(\iota(1)+\rho_{\bar{c}}(\bar{b}) \circ \iota(1)\right)\right) \\
& =\iota^{-1}\left(\iota(a)+\lambda_{\bar{b}}(\bar{c}) \circ\left(\iota(1)+\rho_{\bar{c}}(\bar{b})\right) \circ \iota(1)\right) \\
& =\iota^{-1}\left(\left(\iota(a)+\lambda_{\bar{b}}(\bar{c}) \circ\left(\iota(1)+\rho_{\bar{c}}(\bar{b})\right)\right) \circ \iota(1)\right) \\
& =\iota^{-1}(((\iota(a)+\bar{b} \circ(\iota(1)+\bar{c}))) \circ \iota(1)) \\
& =\iota^{-1}((\iota(a)+\bar{b} \circ(\iota(1)+\bar{c})) \circ \iota(1)),
\end{aligned}
$$

i.e., (11) holds in the left semi-brace $\left(B, \oplus, o^{o p}\right)$. Therefore, the map $r_{B}^{\prime}(a, b):=\left(\lambda_{a}^{\prime}(b), \rho_{b}^{\prime}(a)\right)$ is a solution. Finally, defining $f: B \rightarrow B$ by $f(a)=\bar{a}$, it holds that

$$
\begin{aligned}
r_{B}^{\prime}(f \times f)(a, b) & =r_{B}^{\prime}(\bar{a}, \bar{b})=\left(\lambda_{\bar{a}}^{\prime}(\bar{b}), \rho_{\bar{b}}^{\prime}(\bar{a})\right) \\
& =\left(\overline{\lambda_{a}(b)}, \overline{\rho_{b}(a)}\right)=(f \times f)\left(\lambda_{a}(b), \rho_{b}(a)\right) \\
& =(f \times f) r_{B}(a, b),
\end{aligned}
$$

for all $a, b \in B$, i.e., $r_{B}^{\prime}$ and $r_{B}$ are isomorphic solutions. 
Acknowledgments. The first author is member of GNSAGA (INdAM). The second author is supported by Fonds voor Wetenschappelijk Onderzoek (Flanders), via an FWO Aspirant-mandate.

\section{REFERENCES}

[1] D. Bachiller, F. Cedó, E. Jespers, and J. Okniński. Asymmetric product of left braces and simplicity; new solutions of the YangBaxter equation. Commun. Contemp. Math., 21(8):1850042, 30, 2019.

[2] T. Brzeziński. Towards semi-trusses. Rev. Roumaine Math. Pures Appl., 63(2):75-89, 2018.

[3] T. Brzeziński. Trusses: between braces and rings. Trans. Amer. Math. Soc., 372(6):4149-4176, 2019.

[4] T. Brzeziński. Trusses: Paragons, ideals and modules. Journal of Pure and Applied Algebra, 224(6):106258, 2020.

[5] T. Brzeziński and B. Rybołowicz. Modules over trusses vs modules over rings: direct sums and free modules. arXiv:1909.05807, 2019.

[6] T. Brzeziński and B. Rybołowicz. On congruence classes and extensions of rings with applications to braces. arXiv:1912.00907, 2019.

[7] F. Catino, I. Colazzo, and P. Stefanelli. Semi-braces and the Yang-Baxter equation. J. Algebra, 483:163-187, 2017.

[8] F. Catino, I. Colazzo, and P. Stefanelli. Algebraic tools for Solving the Yang-Baxter equation: Generalized semi-braces and the Yang-Baxter equation. Oberwolfach Reports, 2019(51), 2019.

[9] F. Catino, I. Colazzo, and P. Stefanelli. The matched product of the solutions to the Yang-Baxter equation of finite order. arXiv preprint arXiv:1904.07557, 2019.

[10] F. Catino, I. Colazzo, and P. Stefanelli. Skew left braces with non-trivial annihilator. J. Algebra Appl., 18(2):1950033, $23,2019$.

[11] F. Cedó, T. Gateva-Ivanova, and A. Smoktunowicz. Braces and symmetric groups with special conditions. J. Pure Appl. Algebra, 222(12):3877-3890, 2018.

[12] F. Cedó, E. Jespers, and J. Okniński. Braces and the Yang-Baxter equation. Comm. Math. Phys., 327(1):101-116, 2014.

[13] F. Cedó, E. Jespers, and C. Verwimp. Structure monoids of set-theoretic solutions of the Yang-Baxter equation. arXiv:1912.09710, 2019.

[14] F. Cedó, A. Smoktunowicz, and L. Vendramin. Skew left braces of nilpotent type. Proc. Lond. Math. Soc. (3), 118(6):1367-1392, 2019.

[15] V. G. Drinfel'd. On some unsolved problems in quantum group theory. In Quantum groups (Leningrad, 1990), volume 1510 of Lecture Notes in Math., pages 1-8. Springer, Berlin, 1992.

[16] L. Guarnieri and L. Vendramin. Skew braces and the Yang-Baxter equation. Math. Comp., 86(307):2519-2534, 2017.

[17] E. Jespers, L. Kubat, A. Van Antwerpen, and L. Vendramin. Factorizations of skew braces. Math. Ann., 375(3-4):1649-1663, 2019.

[18] E. Jespers, Ł. Kubat, A. Van Antwerpen, and L. Vendramin. Radical and weight of skew braces and their applications to structure groups of solutions of the Yang-Baxter equation. arXiv:2001.10967, 2020.

[19] E. Jespers and A. Van Antwerpen. Left semi-braces and solutions of the Yang-Baxter equation. Forum Math., 31(1):241-263, 2019.

[20] A. Konovalov, A. Smoktunowicz, and L. Vendramin. On skew braces and their ideals. Experimental Mathematics, pages 1-10, 2018.

[21] V. Lebed and L. Vendramin. Cohomology and extensions of braces. Pacific J. Math., 284(1):191-212, 2016.

[22] M. M. Miccoli. Almost semi-braces and the Yang-Baxter equation. Note Mat., 38(1):83-88, 2018.

[23] W. Rump. Braces, radical rings, and the quantum Yang-Baxter equation. J. Algebra, 307(1):153-170, 2007.

(I. Colazzo) Department of Mathematics, Vrije Universiteit Brussel, Pleinlaan 2, 1050 Brussel

E-mail address: ilaria.colazzo@vub.be

(A. Van Antwerpen) Department of Mathematics, Vrije Universiteit Brussel, Pleinlaan 2, 1050 Brussel

E-mail address: arne.van.antwerpen@vub.be 